\title{
Synthesis and Characterization of Tetrachloro-1,3-Oxazepine Derivatives and Evaluation of their Biological Activities
}

\author{
Abdullah Hussein Kshash \\ Department of Chemistry, Education College for Pure Science, University Of Anbar, \\ 31001, Ramadi, Anbar, Iraq \\ *Corresponding author: E-mail: drabdullahkshash@gmail.com \\ Tel: $+964-7830818171$
}

Received: 05-16-2019

\begin{abstract}
6,7,8,9-Tetrachloro[1,3] oxazepine-1,5-dione derivatives $\mathbf{1 b}$-10b have been synthesized by reacting Schiff bases 1a-10a with tetrachlorophthalic anhydride (TCPA) under $(2+5 \rightarrow 7)$ cycloaddition reaction conditons. All reactions had been monitored using TLC. FT IR and melting points have been used to characterize the Schiff bases; oxazepine compounds 1b-10b were characterized using FT IR, ${ }^{1} \mathrm{H}$ NMR and their melting points. Biological activity for oxazepine compounds has been evaluated against bacterial types (Staphylococcus aureus, Escherichia coli, Klebsiella spp.) and against a fungus (Geotrichum spp.). Variable activities have been observed against used strains of bacteria and fungi.
\end{abstract}

Keywords: Oxazepine, tetrachlorophthalic anhydride, Antibacterial activity, Antifungal activity.

\section{Introduction}

Lately, different heterocyclic compounds were synthesized, using reactions of Schiff bases with various reagents, such as oxazepines. Oxazepines are heterocyclic unsaturated compounds, incorporating two hetero atoms (oxygen and nitrogen). There are many synthetic strategies for synthesis of oxazepines, using gold-, palladium- and rhodium-based catalysts, ${ }^{1-3}$ under microwave irradiation, ${ }^{4}$ and under catalyst-free conditions ${ }^{5}$ as well; all these strategies leading to the formation of 1,3-oxazepines-4,7-diones. Oxazepines were synthesized to be evaluated for their potential activities, e.g. antioxidant and anti-inflammatory activity, ${ }^{6}$ as neuroleptic agents, ${ }^{7}$ kinase inhibitors, ${ }^{8}$ antimicrobial agents. ${ }^{9}$ This paper describes 1,3-oxazepine-4,7-dione derivatives, synthesized by reactions of Schiff bases with TCPA, to provide information of synthetic method and the possible use of 6,7,8,9-tetrachloro[1,3] oxazepine-1,5-dione as an antibacterial (S. aureus, E. coli, Klebsiella spp.) and antifungal agent (Geotrichum spp.), in contrast to other routes which use unsubstituted phthalic anhydride for synthesis of 1,3-oxazepines-1,5-dione.

\section{Experimental Section}

\section{1. Materials}

Aromatic aldehydes, amines and TCPA were supplied from Sigma-Aldrich Chemical Co. used without further purification. Solvents were supplied from Romil. Instrumentation: infrared spectra were recorded as ATR technique on Bruker-Tensor 27 spectrometer. ${ }^{1} \mathrm{H}$ NMR spectra were recorded on Bruker $300 \mathrm{MHz}$ spectrometer using DMSO- $d_{6}$ as the solvent and TMS as the internal standard.

\section{2. Procedure for the Preparation of Schiff Bases 1a-10a}

To a $100 \mathrm{~mL}$ round bottomed flask containing $25 \mathrm{~mL}$ absolute ethanol, aromatic aldehyde $(4.5 \mathrm{mmol})$ and 3 drops of glacial acetic acid, equipped with condenser and stirring bar, aromatic amine $(4.5 \mathrm{mmol})$ dissolved in absolute ethanol $(10 \mathrm{~mL})$ was added. The reaction mixture was refluxed for $3 \mathrm{~h}$, then cooled down to room temperature. Product was collected as a solid by filtration and recrystallized from ethanol. 


\section{3. Characterization of Schiff Bases 1a-10a}

1-(4-Bromophenyl)- $N$-(para-tolyl)methanimine (1a). White solid, yield $1.01 \mathrm{~g}$ (82\%); m.p. $142-144^{\circ} \mathrm{C}$, IR (ATR) $v 3050$ (C-H aromatic), 3025 ( $v$ NC-H), $2982\left(v\right.$ C-H $\left.\mathrm{H}_{\mathrm{al}}\right)$, $1617(v \mathrm{C}=\mathrm{N}), 1609,1502$ (aromatic ring) $\mathrm{cm}^{-1}$.

1-(4-Chlorophenyl)- $\boldsymbol{N}$-(para-tolyl)methanimine (2a). ${ }^{10}$ Beige solid, yield $0.9 \mathrm{~g}$ (88\%); m.p. $125-127^{\circ} \mathrm{C}$ (lit. 124$125^{\circ} \mathrm{C}$ ), IR (ATR) v 3055 (C-H aromatic), 3026 ( $v$ NC-H), $2976\left(v \mathrm{C}-\mathrm{H}_{\mathrm{al}}\right), 1623(\boldsymbol{v} \mathrm{C}=\mathrm{N}), 1587,1499(\mathrm{C}=\mathrm{C}$ aromatic $)$ $\mathrm{cm}^{-1}$.

N-(4-Bromophenyl)-1-(4-chlorophenyl)methanimine (3a). ${ }^{11}$ White solid, yield $1.13 \mathrm{~g}$ (85\%); m.p. $120-123{ }^{\circ} \mathrm{C}$ (lit. $122-124^{\circ} \mathrm{C}$ ), IR (ATR) $v 3063(\mathrm{C}-\mathrm{H}$ aromatic), 3027 ( $v$ $\mathrm{NC}-\mathrm{H}), 1617$ ( $\boldsymbol{v} \mathrm{C}=\mathrm{N}), 1591,1566(\mathrm{C}=\mathrm{C}$ aromatic $) \mathrm{cm}^{-1}$.

N-(4-Bromophenyl)-1-(2,4-dichlorophenyl)methanimine (4a). White solid, yield $1.32 \mathrm{~g}$ (89\%); m.p. 136-138 ${ }^{\circ} \mathrm{C}$, IR (ATR) v 3062 (C-H aromatic), 3014 ( $v$ NC-H), 1612 $(v \mathrm{C}=\mathrm{N}), 1585,1482(\mathrm{C}=\mathrm{C}$ aromatic $) \mathrm{cm}^{-1}$.

N-(4-Bromophenyl)-1-(para-tolyl)methanimine (5a). ${ }^{12}$ White solid, yield $0.93 \mathrm{~g}$ (75\%); m.p. $130-132{ }^{\circ} \mathrm{C}$ (lit. 136.3 $\left.{ }^{\circ} \mathrm{C}\right), \quad$ IR (ATR) $v 3050$ (C-H aromatic), $3023 \quad(v$ $\mathrm{NC}-\mathrm{H}), 2972\left(v \mathrm{C}-\mathrm{H}_{\mathrm{al}}\right), 1621(v \mathrm{C}=\mathrm{N}), 1566,1473(\mathrm{C}=\mathrm{C}$ aromatic) $\mathrm{cm}^{-1}$.

$\mathrm{N}$-(4-Chlorophenyl)-1-(2,4-dichlorophenyl)methanimine (6a). White solid, yield 1.09 g (80\%); m.p. 128-130 ${ }^{\circ} \mathrm{C}$, IR (ATR) v 3062 (C-H aromatic), 3021 ( $v$ NC-H), 1613 $(v \mathrm{C}=\mathrm{N}), 1575,1484(\mathrm{C}=\mathrm{C}$ aromatic $) \mathrm{cm}^{-1}$.

N,1-Bis(4-chlorophenyl)methanimine (7a). ${ }^{10}$ Pale green solid, yield 0.86 g (76\%); m.p. $110-112{ }^{\circ} \mathrm{C}$ (lit. 110-111 $\left.{ }^{\circ} \mathrm{C}\right)$, IR (ATR) v 3056 (C-H aromatic), 3022 ( $v$ NC-H), $1622(v \mathrm{C}=\mathrm{N}), 1591,1486(\mathrm{C}=\mathrm{C}$ aromatic $) \mathrm{cm}^{-1}$.

$\mathrm{N}$-(4-Chlorophenyl)-1-(para-tolyl)methanimine (8a). ${ }^{13}$ Beige solid, yield $0.76 \mathrm{~g}$ (73\%); m.p. $109-111{ }^{\circ} \mathrm{C}$ (lit. 110 $\left.{ }^{\circ} \mathrm{C}\right)$, IR (ATR) v 3052 (C-H aromatic), 3026 ( $v$ NC-H), $2986\left(v \mathrm{C}-\mathrm{H}_{\mathrm{al}}\right), 1620(v \mathrm{C}=\mathrm{N}), 1565,1474(\mathrm{C}=\mathrm{C}$ aromatic $)$ $\mathrm{cm}^{-1}$.

N-(4-Chlorophenyl)-1-(4-methoxyphenyl)methanimine (9a). ${ }^{13}$ Beige solid, yield $0.81 \mathrm{~g}(73 \%)$; m.p. $95-97^{\circ} \mathrm{C}$ (lit. $\left.95{ }^{\circ} \mathrm{C}\right)$, IR (ATR) v 3071 (C-H aromatic), $3018(v$ $\mathrm{NC}-\mathrm{H}), 2963\left(v \mathrm{C}-\mathrm{H}_{\mathrm{al}}\right), 1619(v \mathrm{C}=\mathrm{N}), 1566,1475(\mathrm{C}=\mathrm{C}$ aromatic) $\mathrm{cm}^{-1}$.

1-(4-Bromophenyl)- $\mathrm{N}$-(4-chlorophenyl)methanimine (10a). ${ }^{10}$ Beige solid, yield $1.04 \mathrm{~g}$ (78\%); m.p. $122-124{ }^{\circ} \mathrm{C}$ (lit. $119-120^{\circ} \mathrm{C}$ ), IR (ATR) v 3064 (C-H aromatic), 3025 (v NC-H), $1617(v \mathrm{C}=\mathrm{N}), 1585,1482(\mathrm{C}=\mathrm{C}$ aromatic), $\mathrm{cm}^{-1}$.

\section{4. Procedure for the Synthesis of 1,3- Oxazepines-4,7-dione derivatives $1 \mathrm{~b}-10 \mathrm{~b}$}

To a $100 \mathrm{~mL}$ round bottomed flask containing $25 \mathrm{~mL}$ dry benzene and Schiff bases $\mathbf{1 a}-\mathbf{1 0 a}(1 \mathrm{mmol})$ equipped with condenser, was added TCPA $(1 \mathrm{mmol})$ dissolved in 20 $\mathrm{mL}$ of dry benzene. The reaction mixture was refluxed for $5 \mathrm{~h}$, then stirred overnight at room temperature. Thereafter, the solid product was collected by filtration and recrystallized from ethanol.

\section{5. Characterization of Schiff Bases $1 b-10 b$}

3 - (4-Bromophenyl) - 6, 7,8,9-tetrachloro-4-( $p a-$ ra-tolyl)-3,4-dihydrobenzo[ 1,3$]$ oxazepine-1,5-dione (1b). Pale yellow solid, yield $0.68 \mathrm{~g}$ (66\%); m.p. 178-180 ${ }^{\circ} \mathrm{C},{ }^{1} \mathrm{H}$ NMR $\left(300 \mathrm{MHz}, \mathrm{DMSO}-d_{6}\right) \delta 7.87(\mathrm{~d}, J=8.5 \mathrm{~Hz}$, $\left.2 \mathrm{H}, \mathrm{H}_{11,13}\right), 7.73\left(J=8.4 \mathrm{~Hz}, 2 \mathrm{H}, \mathrm{H}_{2,6}\right), 7.49(\mathrm{~d}, J=8.4 \mathrm{~Hz}$, $\left.2 \mathrm{H}, \mathrm{H}_{10,14}\right), 7.22\left(\mathrm{~s}, 1 \mathrm{H}, \mathrm{H}_{8}\right), 7.16\left(\mathrm{~d}, J=8.3 \mathrm{~Hz}, 2 \mathrm{H}, \mathrm{H}_{3,5}\right)$, $2.28\left(\mathrm{~s}, 3 \mathrm{H}, \mathrm{CH}_{3}\right)$. IR (ATR) $\vee 3045(\mathrm{C}-\mathrm{H}$ aromatic), 1726 $\left(v \mathrm{C}=\mathrm{O}_{\text {lacton }}\right), 1667\left(\vee \mathrm{C}=\mathrm{O}_{\text {lactam }}\right), 1511(\mathrm{CO}-\mathrm{N}), 1321$ $(\mathrm{CO}-\mathrm{O}) \mathrm{cm}^{-1}$.

6,7,8,9-Tetrachloro-3-(4-chlorophenyl)-4-(para -tolyl)-3,4-dihydrobenzo[1,3] oxazepine-1,5-dione (2b). Pale yellow solid, yield $0.71 \mathrm{~g}(63 \%)$; m.p. $179-181{ }^{\circ} \mathrm{C},{ }^{1} \mathrm{H}$ NMR (300 MHz, DMSO- $\left.d_{6}\right) \delta 7.95(\mathrm{~d}, J=8.5 \mathrm{~Hz}, 2 \mathrm{H}$, $\left.\mathrm{H}_{11,13}\right), 7.59$ (d, $\left.J=8.5 \mathrm{~Hz}, 2 \mathrm{H}, \mathrm{H}_{2,6}\right), 7.54-7.44(\mathrm{~m}, 2 \mathrm{H}$, $\left.\mathrm{H}_{10,14}\right), 7.21\left(\mathrm{~s}, 1 \mathrm{H}, \mathrm{H}_{8}\right), 7.17\left(\mathrm{~d}, J=8.2 \mathrm{~Hz}, 2 \mathrm{H}, \mathrm{H}_{3,5}\right), 2.28$ $\left(\mathrm{s}, 3 \mathrm{H}, \mathrm{CH}_{3}\right.$ ). IR (ATR) $\vee 3053$ (C-H aromatic), 1726 $\left(v \mathrm{C}=\mathrm{O}_{\text {lacton }}\right), 1666\left(v \mathrm{C}=\mathrm{O}_{\text {lactam }}\right), 1509(\mathrm{CO}-\mathrm{N}), 1321$ $(\mathrm{CO}-\mathrm{O}) \mathrm{cm}^{-1}$.

4-(4-Bromophenyl)-6,7,8,9-tetrachloro-3-(4-chlorophenyl)-3,4-dihydrobenzo $[1,3]$ oxazepine-1,5-dione (3b). Pale green solid, yield 0.55 g (55\%); m.p. $290{ }^{\circ} \mathrm{C}$ dec., ${ }^{1} \mathrm{H}$ NMR (300 MHz, DMSO- $\left.d_{6}\right) \delta 7.97-7.91(\mathrm{~m}, 2 \mathrm{H}$, $\left.\mathrm{H}_{3,5}\right), 7.76\left(\mathrm{~d}, J=8.7 \mathrm{~Hz}, 2 \mathrm{H}, \mathrm{H}_{11,13}\right), 7.67(\mathrm{~d}, J=8.4 \mathrm{~Hz}$, $\left.2 \mathrm{H}, \mathrm{H}_{2,6}\right), 7.28-7.19\left(\mathrm{~m}, 2 \mathrm{H}, \mathrm{H}_{10,14}\right), 7.21$ (s, $\left.1 \mathrm{H}, \mathrm{H}_{8}\right) . \mathrm{IR}$ $(\mathrm{ATR}) \vee 3063(\mathrm{C}-\mathrm{H}$ aromatic $), 1717\left(\vee \mathrm{C}=\mathrm{O}_{\text {lacton }}\right), 1669$ $\left(v \mathrm{C}=\mathrm{O}_{\text {lactam }}\right), 1541(\mathrm{CO}-\mathrm{N}), 1312(\mathrm{CO}-\mathrm{O}) \mathrm{cm}^{-1}$.

4-(4-Bromophenyl)-6,7,8,9-tetrachloro-3-(2,4-dichlorophenyl)-3,4-dihydrobenzo $[1,3]$ oxazepine-1,5-dione (4b). White solid, yield $0.49 \mathrm{~g}(52 \%)$; m.p. $>300{ }^{\circ} \mathrm{C},{ }^{1} \mathrm{H}$ NMR $\left(300 \mathrm{MHz}\right.$, DMSO- $\left.d_{6}\right) \delta 8.16(\mathrm{~d}, J=8.5 \mathrm{~Hz}, 2 \mathrm{H}$, $\mathrm{H}_{3,5}$ ), 7.81-7.73 (m, 2H, $\left.\mathrm{H}_{2,6}\right), 7.57$ (s, $\left.1 \mathrm{H}, \mathrm{H}_{11}\right), 7.37$ (s, $\left.1 \mathrm{H}, \mathrm{H}_{8}\right), 7.17-7.14\left(\mathrm{~m}, \mathrm{H}, \mathrm{H}_{13}\right), 6.56(\mathrm{~d}, J=8.6 \mathrm{~Hz}, 8 \mathrm{H}$, $\left.\mathrm{H}_{14}\right)$. IR (ATR) v 3060 (C-H aromatic), $1720\left(v \mathrm{C}=\mathrm{O}_{\text {lacton }}\right)$, $1670\left(v \mathrm{C}=\mathrm{O}_{\text {lactam }}\right), 1532(\mathrm{CO}-\mathrm{N}), 1313(\mathrm{CO}-\mathrm{O}) \mathrm{cm}^{-1}$.

4-(4-Bromophenyl)-6,7,8,9-tetrachloro-3-(para -tolyl)-3,4-dihydrobenzo[1,3] oxazepine-1,5-dione (5b). Yellow solid, yield $0.691 \mathrm{~g}$ (77\%); m.p. $260{ }^{\circ} \mathrm{C} \mathrm{dec} .,{ }^{1} \mathrm{H}$ NMR (300 MHz, DMSO- $\left.d_{6}\right) \delta 7.88-7.81\left(\mathrm{~m}, 2 \mathrm{H}, \mathrm{H}_{3,5}\right)$, 7.63-7.56 (m, 2H, $\left.\mathrm{H}_{2,6}\right), 7.23\left(\mathrm{~d}, J=2.1 \mathrm{~Hz}, 1 \mathrm{H}, \mathrm{H}_{8}\right), 7.15$ 
(d, $\left.J=8.7 \mathrm{~Hz}, 2 \mathrm{H}, \mathrm{H}_{10,14}\right), 6.58-6.51\left(\mathrm{~m}, 2 \mathrm{H}, \mathrm{H}_{11,13}\right), 2.38$ (s, 3H, $\mathrm{CH}_{3}$ ). IR (ATR) v 3047 (C-H aromatic), 1721 ( $\left.v \mathrm{C}=\mathrm{O}_{\text {lacton }}\right), 1668$ ( $\left.\vee \mathrm{C}=\mathrm{O}_{\text {lactam }}\right), 1542(\mathrm{CO}-\mathrm{N}), 1314$ $(\mathrm{CO}-\mathrm{O}) \mathrm{cm}^{-1}$.

6,7,8,9-Tetrachloro-4-(4-chlorophenyl)-3-(2,4-dichlorophenyl)-3,4-dihydrobenzo $[1,3]$ oxazepine-1,5-dione (6b). Yellow solid, yield 0.64 g (64\%); m.p. > $300{ }^{\circ} \mathrm{C},{ }^{1} \mathrm{H}$ NMR (300 MHz, DMSO- $\left.d_{6}\right) \delta 7.70-7.57\left(\mathrm{~m}, 2 \mathrm{H}, \mathrm{H}_{3,5}\right)$, 7.48-7.41 (m, 2H, $\left.\mathrm{H}_{2,6}\right), 7.37$ (s, $\left.1 \mathrm{H}, \mathrm{H}_{11}\right), 7.30$ (s, $1 \mathrm{H}, \mathrm{H}_{8}$ ), $7.06\left(\mathrm{~d}, J=8.6 \mathrm{~Hz}, 1 \mathrm{H}, \mathrm{H}_{13}\right), 6.62\left(\mathrm{~d}, J=8.6 \mathrm{~Hz}, 1 \mathrm{H}, \mathrm{H}_{14}\right)$. IR (ATR) v 3062 (C-H aromatic), $1722\left(v \mathrm{C}=\mathrm{O}_{\text {lacton }}\right), 1667$ $\left(v \mathrm{C}=\mathrm{O}_{\text {lactam }}\right), 1490(\mathrm{CO}-\mathrm{N}), 1312(\mathrm{CO}-\mathrm{O}) \mathrm{cm}^{-1}$.

6,7,8,9-Tetrachloro-3,4-bis(4-chlorophenyl)-3,4-dihydrobenzo $[1,3]$ oxazepine-1,5-dione $(7 \mathbf{b})$. Pale yellow solid, yield $0.78 \mathrm{~g}$ (73\%); m.p. $288-290{ }^{\circ} \mathrm{C},{ }^{1} \mathrm{H}$ NMR $(300 \mathrm{MHz}$, DMSO- $\left.d_{6}\right) \delta 7.64\left(\mathrm{~d}, J=8.9 \mathrm{~Hz}, 2 \mathrm{H}, \mathrm{H}_{3,5}\right), 7.49-7.39(\mathrm{~m}$, $2 \mathrm{H}, \mathrm{H}_{11,13}$ ), 7.30 (s, $1 \mathrm{H}, \mathrm{H}_{8}$ ), 7.08-6.98 (m, 2H, H $\mathrm{H}_{2,6}$ ), 6.58 $\left(\mathrm{d}, J=8.6 \mathrm{~Hz}, 2 \mathrm{H}, \mathrm{H}_{10,14}\right)$. IR (ATR) v 3063 (C-H aromatic), $1717\left(\vee \mathrm{C}=\mathrm{O}_{\text {lacton }}\right), 1668\left(\vee \mathrm{C}=\mathrm{O}_{\text {lactam }}\right), 1541(\mathrm{CO}-\mathrm{N})$ $1316(\mathrm{CO}-\mathrm{O}) \mathrm{cm}^{-1}$.

6,7,8,9-Tetrachloro-4-(4-chlorophenyl)-3-(para -tolyl)-3,4-dihydrobenzo[1,3] oxazepine-1,5-dione (8b).
Pale yellow solid, yield $0.79 \mathrm{~g}(71 \%)$; m.p. $265-267^{\circ} \mathrm{C},{ }^{1} \mathrm{H}$ NMR $\left(300 \mathrm{MHz}, \mathrm{DMSO}-d_{6}\right) \delta 7.64(\mathrm{~d}, J=8.9 \mathrm{~Hz}, 2 \mathrm{H}$, $\left.\mathrm{H}_{3,5}\right), 7.46-7.41\left(\mathrm{~m}, 2 \mathrm{H}, \mathrm{H}_{2,6}\right), 7.27\left(\mathrm{~s}, 1 \mathrm{H}, \mathrm{H}_{8}\right), 7.06-7.00$ (m, 2H, H $\left.\mathrm{H}_{10,14}\right), 6.57$ (d, $\left.J=8.8 \mathrm{~Hz}, 2 \mathrm{H}, \mathrm{H}_{11,13}\right), 2.38$ (s, $\left.3 \mathrm{H}, \mathrm{CH}_{3}\right)$. IR (ATR) v $3062(\mathrm{C}-\mathrm{H}$ aromatic), 1722 $\left(v \mathrm{C}=\mathrm{O}_{\text {lacton }}\right), 1666\left(\nu \mathrm{C}=\mathrm{O}_{\text {lactam }}\right), 1543(\mathrm{CO}-\mathrm{N}) 1327$ $(\mathrm{CO}-\mathrm{O}) \mathrm{cm}^{-1}$.

6,7,8,9-Tetrachloro-4-(4-chlorophenyl)-3-(4-methoxyphenyl)-3,4-dihydrobenzo $[1,3]$ oxazepine-1,5-dione (9b). Pale green solid, yield $1.07 \mathrm{~g}(74 \%)$; m.p. $272-274^{\circ} \mathrm{C}$, ${ }^{1} \mathrm{H}$ NMR $\left(300 \mathrm{MHz}, \mathrm{DMSO}-d_{6}\right) \delta 7.67-7.60\left(\mathrm{~m}, 2 \mathrm{H}, \mathrm{H}_{3,5}\right)$, $7.44\left(\mathrm{~d}, J=8.9 \mathrm{~Hz}, 2 \mathrm{H}, \mathrm{H}_{2,6}\right), 7.25\left(\mathrm{~s}, 1 \mathrm{H}, \mathrm{H}_{8}\right), 7.08(\mathrm{~d}, J=$ $\left.8.8 \mathrm{~Hz}, 2 \mathrm{H}, \mathrm{H}_{10,14}\right), 6.63-6.53\left(\mathrm{~m}, 2 \mathrm{H}, \mathrm{H}_{11,13}\right), 3.84(\mathrm{~s}, 3 \mathrm{H}$, $\left.\mathrm{OCH}_{3}\right)$. IR (ATR) v 3054 (C-H aromatic), $1723\left(v \mathrm{C}=\mathrm{O}_{\text {lac }-}\right.$ ton), 1667 ( $\left.v \mathrm{C}=\mathrm{O}_{\text {lactam }}\right), 1547(\mathrm{CO}-\mathrm{N}) 1314(\mathrm{CO}-\mathrm{O}) \mathrm{cm}^{-1}$.

3-(4-Bromophenyl)-6,7,8,9-tetrachloro-4-(4-chlorophenyl)-3,4-dihydrobenzo[ 1,3$]$ oxazepine-1,5-dione (10b). Yellow solid, yield 0.58 g (60\%); m.p. $250-253{ }^{\circ} \mathrm{C}$, ${ }^{1} \mathrm{H}$ NMR $\left(300 \mathrm{MHz}, \mathrm{DMSO}-d_{6}\right) \delta 7.88(\mathrm{~d}, J=8.5 \mathrm{~Hz}, 2 \mathrm{H}$, $\mathrm{H}_{11,13}$ ), 7.74 (d, $\left.J=8.5 \mathrm{~Hz}, 2 \mathrm{H}, \mathrm{H}_{3,5}\right), 7.69-7.60$ (m, $2 \mathrm{H}$, $\mathrm{H}_{2,6}$ ), 7.44 (d, $\left.J=8.9 \mathrm{~Hz}, 2 \mathrm{H}, \mathrm{H}_{10,14}\right), 7.30$ (s, 1H, $\mathrm{H}_{8}$ ). IR $($ ATR $) \vee 3062(\mathrm{C}-\mathrm{H}$ aromatic $), 1723\left(v \mathrm{C}=\mathrm{O}_{\text {lacton }}\right), 1667(v$ $\left.\mathrm{C}=\mathrm{O}_{\text {lactam }}\right), 1591(\mathrm{CO}-\mathrm{N}) 1312(\mathrm{CO}-\mathrm{O}) \mathrm{cm}^{-1}$.

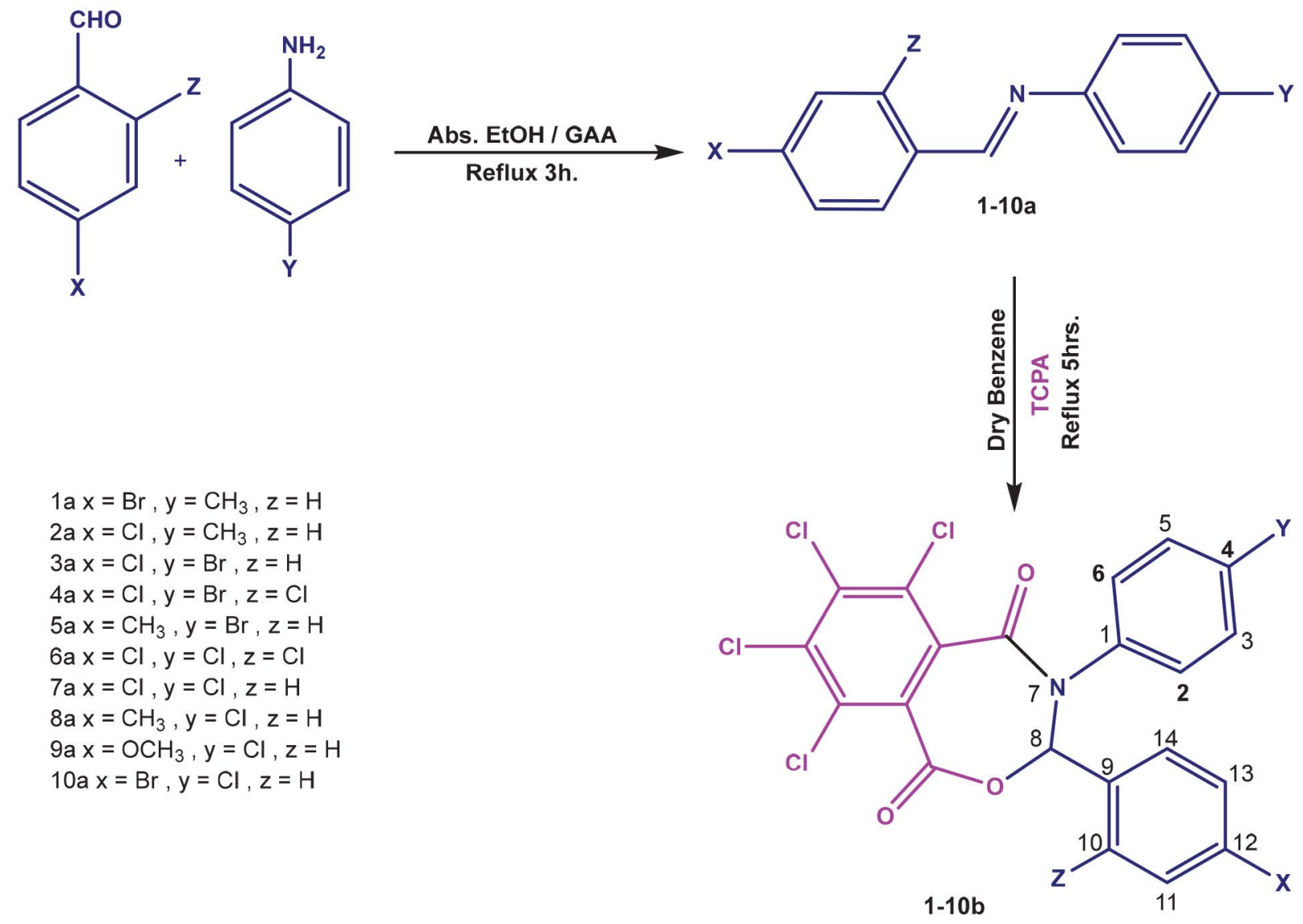

Scheme 1. Synthetic route for synthesis of $\mathbf{1 b}-\mathbf{1 0 b}$ compounds 


\section{Results and Discussion}

\section{1. Chemistry}

Target oxazepine compounds $\mathbf{1 b}-\mathbf{1 0 b}$ were synthesized according to the route presented in Scheme 1.

Imines 1a-10a have been synthesized as precursors for oxazepine compounds by condensation reaction of aromatic aldehydes and primary aromatic amines using absolute ethanol as the solvent. According to the yields obtained, it can be indicated that when the reacting compounds are substituted by electron withdrawing groups (EWG) on benzaldehyde (at the para position), greater amount of the product is obtained than was the case with other compounds, this being due to the substituents having -I effect that increase the magnitude of the positive charge $\left(\delta^{+}\right)$on the carbonyl carbon atom. Hence, increasing the reactivity of benzaldehyde to the attack by amine (as a nucleophile).

Oxazepines have been synthesized by reactions of imines with TCPA, using dry benzene as the solvent, in (2 $+5 \rightarrow 7$ ) cycloaddition reaction. This reactions type is controlled by orbital symmetry. Therefore, the frontier molecular orbitals for the reactants have to be taken into consideration. Moreover, orbitals of the reactants (anhydride and imines) have to overlap in the convenient way to afford oxazepines by suprafacial manner.

Synthesis of oxazepines has taken place by overlapping of orbitals for imines and TCPA, through the formation of a four-membered ring as the transition state; increasing this overlap led to the formation of a seven -membered ring compounds, as depicted in Scheme 2.

In general, electron donating groups (EDG) increase HOMO energy levels, therefore, reduce the required energy to accomplish the oxazepine formation reaction; in this study, the reaction progress was in accord with the pericyclic mechanism where FMO of imines serve as HOMO, whereas those of TCPA as LUMO; this expectation is brought up through the notice that the yield for $\mathbf{5 b}, \mathbf{8 b}$ and $\mathbf{9 b}$ is the highest, due to the precursor imines $5 \mathbf{a}, \mathbf{8 a}$ and $9 \mathbf{a}$ having electron donating groups that increase the HOMO energy level for imines, giving rise to decreasing the required reaction's energy (Figure 1).

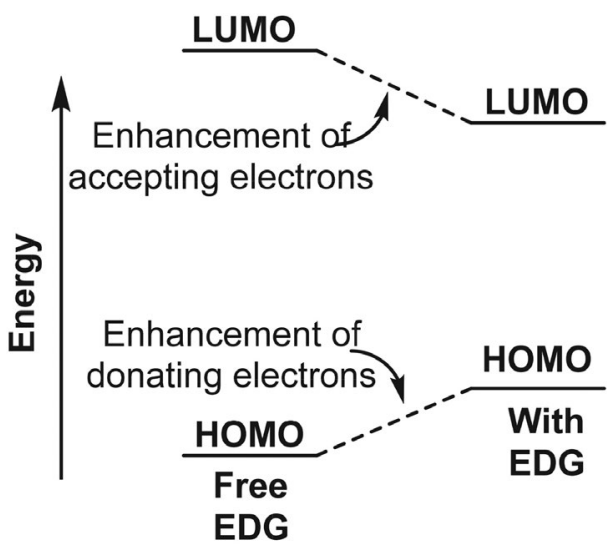

Figure 1. HOMO and LUMO energy level

\section{1. 1. Characterization of Prepared Compounds}

Imine compounds 1a-10a were synthesized by reactions of aromatic aldehydes with amines, using absolute ethanol as the solvent. Color, melting point and $\mathrm{R}_{f}$ changes (TLC analysis) indicated the formation of new compounds, FT IR spectra for compounds $\mathbf{1 a - 1 0 a}$ showed the disappearance of stretching vibration bands for $\mathrm{NH}_{2}$ group for substituted aniline, and $v \mathrm{C}=\mathrm{O}$ group absorption band for substituted benzaldehyde, moreover, appearance of strong vibration band at the range $1612-1623 \mathrm{~cm}^{-1}$ can be attributed to the $v \mathrm{C}=\mathrm{N}$ azomethine group, beside absorption bands at 3050-3071 $\mathrm{cm}^{-1}$ for aromatic $v \mathrm{C}-\mathrm{H}, 3014-$ $3032 \mathrm{~cm}^{-1}$ for $v \mathrm{NC}-\mathrm{H}$ and 1566-1609 and 1473-1566 $\mathrm{cm}^{-1}$ for aromatic rings.

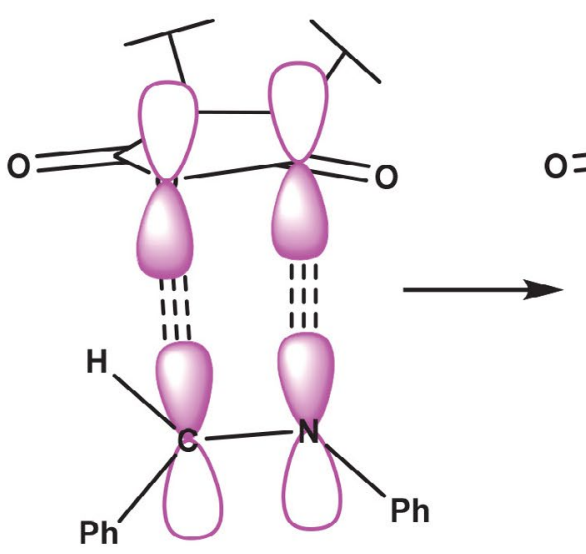

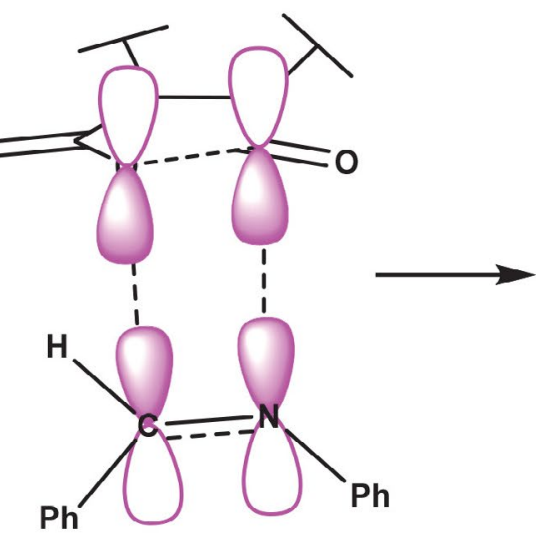

[TS]

Scheme 2. Formation mechanism of oxazepine compounds 
Table 1. In vitro zone of inhibition ( $\mathrm{mm}$ ) of target oxazepine compounds.

\begin{tabular}{|c|c|c|c|c|c|c|c|c|c|c|}
\hline \multirow[t]{2}{*}{ Strains } & \multicolumn{8}{|c|}{ Compound } & \multirow[b]{2}{*}{$9 b$} & \multirow[b]{2}{*}{$10 \mathrm{~b}$} \\
\hline & $\mathbf{1 b}$ & $2 \mathbf{b}$ & $3 \mathbf{b}$ & $4 b$ & $5 b$ & $\mathbf{6 b}$ & $7 \mathbf{b}$ & $8 b$ & & \\
\hline Staphylococcus aureus & 10 & 11 & 15 & 14.5 & 13 & 14 & 13.5 & 14.5 & 14 & 17 \\
\hline Escherichia coli & 8 & 8 & 9 & 8 & 9.5 & 8.5 & 8 & 0 & 0 & 8.5 \\
\hline Klebsiella sp. & 8.5 & 0 & 10 & 10 & 9 & 0 & 0 & 0 & 0 & 0 \\
\hline Geotrichum sp. & 10 & 11 & 14 & 17 & 11.5 & 18 & 18 & 15 & 17 & 19 \\
\hline
\end{tabular}

Oxazepines $\mathbf{1 b}-\mathbf{1 0 b}$ were synthesized by reactions of synthesized imines 1a-10a and TCPA using dry benzene as the solvent in accord with the mechanism described in Scheme 2. FT IR spectra for oxazepine compounds $\mathbf{1 b}-\mathbf{1 0 b}$ showed the disappearance of stretching vibration bands for $v \mathrm{C}=\mathrm{N}$ azomethine group, furthermore, appearance of vibration absorption band at 3045$3063 \mathrm{~cm}^{-1}$ assigned to the aromatic $v \mathrm{C}-\mathrm{H}$, at $1717-1726$ $\mathrm{cm}^{-1}$ for $v \mathrm{C}=\mathrm{O}_{\text {lacton}}, 1666-1670 \mathrm{~cm}^{-1}$ for $v \mathrm{C}=\mathrm{O}_{\text {lactam}}$, $1490-1547 \mathrm{~cm}^{-1}$ for $v$ CO-N and $1312-1327 \mathrm{~cm}^{-1}$ for $v$ $\mathrm{CO}-\mathrm{O}$ was observed.

\section{2. Biological Activity}

\section{2. 1. Antibacterial Activity}

Antibacterial activities for oxazepine compounds 1b-10b were evaluated against Staphylococcus aureus, Escherichia coli and Klebsiella spp. by applying $2 \mathrm{mg} /$ well of the synthesized compounds dissolved in DMSO, then incubated at $37^{\circ} \mathrm{C}$ for $24 \mathrm{~h}$. Results indicate Klebsiella spp. resistance for compounds $\mathbf{6 b}-\mathbf{1 0 b}$, while $E$. coli was resistant to compounds $\mathbf{8 b}$ and $\mathbf{9 b}$, other compounds demonstrated good inhibition against microbial tested strains, inhibition zone data in $\mathrm{mm}$ are given in Table 1.

\section{2. 2. Antifungal Activity}

Antifungal activities for the synthesized oxazepine compounds 1b-10b were screened against Geotrichum spp. by applying $2 \mathrm{mg} /$ well of the synthesized compounds dissolved in DMSO, then incubated at $37^{\circ} \mathrm{C}$ for $24 \mathrm{~h}$. Results show that all of the synthesized compounds demonstrate good inhibition against the tested fungus, and no resistance for oxazepine compounds was found; inhibition zone data in $\mathrm{mm}$ are given in Table 1.

Data of antibacterial and antifungal activity (Table 1) revealed that compounds with substituents $\mathrm{Cl}$ and $\mathrm{Br}$ have the highest inhibition zone, particularly against $S$. aureus and Geotrichum spp., whereas compounds with substituents $\mathrm{CH}_{3}$ and $\mathrm{OCH}_{3}$ have the lowest inhibition, this can be attributed to van der Waals interaction with chloro and bromo substituent compounds; para-Br substituted compounds are more effective than para-Cl substituted compounds due to the size of the bromine atom which facilitates halogen bond interactions by supplying van der Waals radii between the donor and the acceptor atom.

\section{Conclusion}

New tetrachloro[1,3] oxazepine-1,5-diones $\mathbf{1 b}$-10b were synthesized in good yields, had been characterized by different spectroscopic methods, their anti-bacterial biological activity evaluated against Staphylococcus aureus, Escherichia coli, and Klebsiella spp.) and their anti-fungal properties against Geotrichum spp., variable activities were recorded for the tested compounds.

\section{References}

1. K. Goutham, D. A. Kumar, S. Suresh, B. Sridhar, R. Narender, G. V. Karunakar, J. Org. Chem. 2015, 80, 11162-11168. DOI:10.1021/acs.joc.5b01733

2. Y. Wu, C. Yuan, C. Wang, B. Mao, H. Jia, X. Gao, J. Liao, F. Jiang, L. Zhou, Q. Wang, H. Guo, Org. Lett. 2017, 19, 6268-6271.

DOI:10.1021/acs.orglett.7b02704

3. Y. O. Ko, H. J. Jeon, D. J. Jung, U. B. Kim, S. G. Lee, Org. Lett. 2016, $18,6432-6435$.

DOI:10.1021/acs.orglett.6b03328

4. N. I. Taha, Int. J. Org. Chem. 2017, 7, 219-228. DOI:10.4236/ijoc.2017.73016

5. A. H. Kshash, M. G. Mokhlef, Indones. J. Chem. 2017, 17(2), 330-335. DOI:10.22146/ijc.22437

6. A. K. Alexander, L. Joseph, M. George, Eur. J. Pharm. Med. Res. 2016, 3(7), 330-336.

7. K. Bajaj, V. K. Srivastava, A. Kumar, Indones. J. Chem. 2003, 42B, 1149-1155.

8. X. Chen, Y. Du, H. Sun, F. Wang, L. Kong, M. Sun, Bioorg. Med. Chem. Lett. 2014, 24, 884-887.

DOI:10.1016/j.bmcl.2013.12.079

9. J.Tang,Y.-L.Jiang,B.-X.Wang, Y.-M.Shen,Z.Naturforsch.C2014, 69(7-8), 283-290.

DOI:10.5560/znc.2014-0029

10. J. S. Bennett, K. L. Charles, M. R. Miner, C. F. Heuberger, E. J. Spina, M. F. Bartels, T. Foreman, Green Chem. 2009, 11, 166-168. DOI:10.1039/b817379f

11. H. Naeimi, K. Rabiei, J. Chin. Chem. Soc. 2012, 59, 208-212. DOI:10.1002/jccs.201100354

12. L. Jothi, R. R. Babu, K. Ramamurthi, J. Miner. Mater. Char. Eng. 2014, 2, 308-318. DOI:10.4236/jmmce.2014.24036

13. Y. Algamala, R. M. Ghalib, Int. J. Sci. Basic Appl. Res. 2015, 20, 53-76. DOI:10.12659/MSMBR.890835 


\section{Povzetek}

$S$ pomočjo reakcije med Schiffovimi bazami 1a-10a in tetrakloroftalanhidridom (TCPA) smo s pomočjo $(2+5 \rightarrow 7)$ cikloadicije pripravili 6,7,8,9-tetrakloro[1,3] oksazepin-1,5-dionske derivate $\mathbf{1 b}$-10b. Reakcije smo spremljali s TLC. S pomočjo FT IR spektroskopije in določitvijo temperature tališča smo karakterizirali pripravljene Schiffove baze; strukture oksazepinskih spojin $\mathbf{1 b}$-10b smo določili s pomočjo FT IR in ${ }^{1} \mathrm{H}$ NMR ter z določitvijo temperature tališča. Biološke aktivnosti oksazepinskih derivatov smo določili na bakterijah (Staphylococcus aureus, Escherichia coli, Klebsiella spp.) in glivi (Geotrichum spp.). Za različne spojine smo, v odvisnosti od uporabljenega organizma bakterije oz. glive, določili tudi različne aktivnosti. 